\title{
ANÁLISIS ESTRUCTURAL Y SIGNIFICADO LINGÜÍSTICO
}

\author{
José Miguel Rodríguez Zamora
}

\begin{abstract}
RESUMEN
Este artículo estudia el concepto de significado en la semántica estructural. Se pone especial atención al campo semántico y al rasgo. Se propone el concepto de operador semántico como instrumento de análisis metodológico de la estructura semántica.
\end{abstract}

\begin{abstract}
This article studies the concept of meaning in the structural semantics; specially the semantic field and feature. Its proposed the idea of semantic operator as instrument for metodological analysis.
\end{abstract}

\section{Introducción}

En la década de los años 50, los lingüistas, respaldados en buenos argumentos, ya tenían claro que la lengua es un sistema complejo, autónomo y compuesto de elementos interdependientes, es decir, una estructura. Pero una estructura de cierto tipo y con determinadas características. Una de estas notas distintivas es la de que las unidades significativas de la semántica, los semas, adquieren su significado dentro del propio sistema. Es decir, que su significado no es inherente al signo, sino inmanente a la estructura de la lengua (Dubois et al. 1999: 445).

Es posible que esta haya sido una de las contribuciones más relevantes del estructuralismo lingüístico al conocimiento del significado comunicativo. Por ello se puede suscribir la siguiente afirmación de Jakobson:

¿Es que existe alguien hoy día que no conciba la lengua en su conjunto, en sí misma y por sí misma y, a la vez, como parte constituyente de la cultura y de la sociedad? La lingüística se ha convertido en una ciencia bifronte, ocupada permanentemente de la relación entre el todo y sus partes (Jakobson 1974: 1984).

Desde entonces, se han realizado numerosos estudios e investigaciones acerca del significado y la estructura de la lengua. El mismo estructuralismo se ha ramificado en tantas corrientes que en ocasiones resulta casi irreconocible (Marín 1994: 56). Sin embargo, el tema del 
significado lingüístico aún está lejos de ser totalmente resuelto. Todo lo contrario, en la actualidad parece aún más complejo y cada vez más relevante para la linguiística (Alcaráz Varó 1997: 517). ¿Cómo se construye el significado linguiístico?¿Qué función desempeña el análisis estructural en este proceso? En el presente artículo se propondrá una vía de solución a este problema.

\section{Análisis componencial y rasgo semántico}

De acuerdo con la semántica estructural, cada unidad lingüística significativa puede, a su vez, subdividirse en unidades menores llamadas rasgos (Crystal 1997: 100-2). De esta forma, el significado de una palabra se puede conocer al analizar sus rasgos componentes, o sea, las partes y elementos que conforman las propiedades de significado. De ahí que este significado se derive de los rasgos de oposiciones y semejanzas que establecen las unidades mínimas. Más bien podría decirse que los mismos son fragmentos del todo constituido por el significado. Sin embargo, el rasgo no sólo es significativo al nivel de las microestructuras componenciales sino que abarca el conjunto del texto (Wotjak 1979: 269).

Derivado de la lingüística estructural y en correspondencia con su método de investigación surge el análisis componencial, el cual concierne al estudio o análisis (a-nálisis: descomposición) de los rasgos del significado. Es una consecuencia lógica de los presupuestos de la propia lingüística estructural. Por eso, el análisis componencial se puede entender como "un medio de formalizar o hacer absolutamente precisas las relaciones de sentido que los lexemas mantienen entre sí. Como el mismo nombre lo indica, dichos análisis consisten en la descomposición del sentido de un lexema en sus elementos constituyentes" (Lyons 1997: 134). Estos elementos constituyentes o rasgos no se derivan ni de las funciones o taxonomías morfo-sintácticas (verbo, sustantivo, adjetivo, etc.), ni de los accidentes del nombre o flexiones nominales (caso nominativo, dativo, acusativo, etc.). Más bien poseen características propias.

Según Berruto, los rasgos pueden ser considerados como "entidades de significado mínimas, presentes en el significado de muchas palabras, y que combinándose simultáneamente, forman el significado de una palabra, distinguiéndolo del de todas las otras. Son llamados "átomos del significado" (Berruto 1974: 121-2). Por este motivo, se puede afirmar que el significado de toda palabra surge al descomponerse en unidades menores; este es un hecho propio, inherente al propio proceso de significación según la semántica estructural ${ }^{1}$.

Es importante destacar que la noción epistemológica subyacente consiste en la idea de la diferenciación de los miembros semánticamente significativos. La categoría de diferenciación prescribe la función propia de la unidad diferenciadora. Es decir, que cada unidad de significado tiene características propias que la hacen distinta de otras unidades semánticas. Como ya había señalado de Saussure, la materia lingüística no es homogénea ni indiferenciada. Esta propiedad ontológicamente distintiva es la que permite constituir la base diferencial y también es la que posibilita tanto el análisis como la comparación metodológica de unidades diversas. Recuérdese que el modelo que utiliza la semántica estructural proviene de la fonología, en la cual tanto las unidades semánticas como las fonéticas son susceptibles de descomposición sistemática. Según Lyons, la lengua particular establece un subconjunto con las posibilidades potenciales de descomposición de las unidades; es un procedimiento analítico. En efecto, este autor afirma: 
Se trata de la noción de que los fonemas y los significados de las palabras de todas las lenguas son analizables en componentes todavía menores (o rasgos distintivos) y que, aunque los componentes (esto es, los fonemas y los significados de palabras) y sus relaciones sintagmáticas y paradigmáticas sean exclusivas para cada lengua, los componentes últimos del análisis fónico y significativos son lingüísticamente neutrales (en el sentido de que su entidad es ajena a la lengua en cuestión). De acuerdo con esta postura, ni la sustancia fónica ni la sustancia semántica constituyen un continuo indiferenciado, en cuyo interior las lenguas sientan distinciones puramente arbitrarias. Lo que hay en cada caso, se afirma, es un conjunto de distinciones potenciales, del cual cada lengua realiza un subconjunto (Lyons 1989: 231).

Sin embargo, el territorio no está completamente despejado, como a primera vista podría parecer. Más bien, la idea de descomposición y de su eventual composicionalidad suscita numerosas cuestiones las cuales no siempre son fáciles de explicar. La primera de ellas es de naturaleza ontológica, y es la siguiente: ¿existen los rasgos en la realidad o es una invención heurística del investigador? La segunda es epistemológica: ¿cómo se consigue el reconocimiento de los rasgos? Para dar respuesta a estas cuestiones, se comenzará con la última.

Un camino que se sigue con frecuencia se puede expresar de la siguiente forma: cuando se tienen varios términos que poseen algo en común en razón de su significado, es decir, que comparten un mismo campo semántico, se procede a determinar lo que diferencia a los significados de los distintos términos. Es un proceso de diferenciación inicial derivado de un reconocimiento de semejanzas de los rasgos semánticos. Luego se procede a establecer ecuaciones proporcionales; ej: hombre-mujer-niño. La conclusión que se extrae es que lo que estos grupos de palabras tienen en común constituye un componente semántico. Como es evidente, no todos los rasgos constituyen un mismo tipo. Ciertamente, existen diversos tipos de rasgos, los prosódicos, los paralingüísticos, entre otros. Sin embargo, para la semanticidad lo esencial es el rasgo propiamente semántico (Lyons 1997: 37). Algunos suponen solo un objeto al que se refieren; son llamados "rasgos de un sólo lugar" y son los lexemas simples. Otros, por el contrario, implican dos o más objetos entre los que se establece una relación, llamados "rasgos de relación"; son los "rasgos de dos o varios lugares" o lexemas compuestos (Lyons 1989: 463-8).

El lexema es la unidad que trasporta el significado referencial. Este hecho permite un principio de clasificación ya que los lexemas pueden organizarse en clases o grupos significativos, cuyos rasgos permiten su ubicación en un lugar o en varios lugares semánticos ${ }^{2}$. Por ejemplo, "hombre" es un lexema propio de un solo lugar, en tanto que "padre" o "madre" son de dos lugares porque tienen el rasgo de relación "ascendiente"; no existe padre o madre sin un hijo, etc. ${ }^{3}$ En realidad, este procedimiento funciona debido a un operador de negación, es decir, a una eliminación de rasgos por sustitución de elementos constituyentes. Como un complemento a este operador negativo se encuentra la función compositiva de los rasgos, la cual implica tanto el valor de los constituyentes como las reglas operativas ${ }^{4}$ (Lyons 1997: 138).

Un aspecto interesante y de extraordinaria importancia es el hecho de que los rasgos poseen propiedades tanto sintácticas como semánticas dentro del sistema de una determinada lengua. ¿Cuáles son estas propiedades? En general, se puede afirmar que las primeras corresponden a las que especifican las características de las palabras que les permiten combinarse selectivamente con otras; en este sentido, forman parte de una estructura formal regida por reglas de combinación. Este procedimiento actualiza mentalmente los cálculos que el hablanteoyente establece para organizar los elementos de forma selectiva. La idea de cálculo expresa la facultad psicolingüística que posee el hablante-oyente para establecer la funcionalidad gramatical de acuerdo con unidades normativas ${ }^{5}$. 
Unido a este proceso surge el de interordinación. Este concepto hace referencia al ámbito más allá de las reglas de combinación sintáctica, pues se dirige hacia la especificación de la forma en que los elementos gramaticales se combinan con otros para establecer significados; con ello consigue que se identifique la naturaleza semántica de estas. Como es sabido, en este proceso también interviene una dimensión pragmática que determina la propiedad o pertenencia a registros particulares y su frecuencia de uso.

Asimismo, se afirma que el estudio de los rasgos semánticos, sintácticos y aún los pragmáticos permite explicar la distinción entre significado léxico (semántico) y significado gramatical (normativo), además de la que existe entre denotación y connotación. La conclusión a la que se llega necesariamente es a la primera regla del análisis componencial: todo significado, en principio, se puede representar mediante un conjunto de rasgos ${ }^{6}$. Esto implica que el significado se determina por la conjunción de un grupo de rasgos distintivos unidos por medio de características diferenciales dentro de un mismo campo semántico de naturaleza interordinal. Pero esta vinculación puede revestir diversa naturaleza, de tal manera que existe una relación ya sea de independencia o de dependencia, cuando un rasgo implica necesariamente a otro. Las consecuencias de este hecho remiten a la respuesta a la segunda pregunta de naturaleza ontológica; es decir, a la reflexión sobre la existencia de los rasgos semánticos. Pero aún conviene analizar otros patrones.

Una constatación elemental puede ser ilustrativa. Dados dos rasgos, x-y, ocurre la dependencia cuando al afirmar x se afirma simultáneamente y. En otras palabras, x depende de y. Esto sucede porque siempre existe una red de dependencia de los rasgos; ej: hombre: + humano; vegetal: -humano, etc. Son rasgos de dos espacios o de dos lugares ya sea por afirmación o por la vía de la negación.

Por otra parte, las relaciones de oposición suceden cuando dos rasgos son incompatibles entre sí: + macho: -macho. En este caso, la representación en rasgos del significado de la palabra no puede incluir rasgos que se opongan entre sí. En este punto, el estructuralismo sigue el clásico principio de no contradicción. Otro tipo de relación posible es el de solidaridad, que se presenta cuando un término tiene como rasgo sintáctico el rasgo semántico de otro término. Ej.: anciano con persona. Una importante consecuencia de lo anterior es la siguiente: debido a la naturaleza de las relaciones entre los rasgos es que se puede, en principio, reformular en términos de rasgos las relaciones entre los lexemas. De esta forma, la definición del campo semántico puede ser vista desde el enfoque del análisis en rasgos semánticos, lo cual permite concluir que un campo semántico se conforma con los términos que tienen un conjunto de rasgos semánticos en común. En otras palabras, el campo semántico se deriva de las relaciones establecidas por un conjunto de rasgos llamados matrices de rasgos (Alcaráz Varó 1997: 346).

Sin embargo, debe advertirse que los términos que constituyen un mismo campo semántico pertenecen todos a la misma clase gramatical o parte del discurso ${ }^{7}$. Estos elementos constituyen la base para la respuesta sobre la propia naturaleza de los rasgos. En este caso, se trata de determinar el valor ontológico de la realidad objetiva de los rasgos, o si, por el contrario, los rasgos son creaciones subjetivas. Es decir, admitiendo que existan los rasgos, se trata de determinar si estos actúan en un nivel sub-semántico o sub-lexemático. Está también el problema de la naturaleza perifrástica de los rasgos, pues se aduce que el estudio de los rasgos corresponde a un segundo nivel, ya que es un metalenguaje sobre la propia lengua de la significación semántica. Se suele afirmar que las palabras de las que se estudia el significado son entidades de la lengua, mientras que los rasgos son entidades de la metalengua, e, incluso, de 
un posible metalenguaje universal (Lyons 1989: 312). De otra forma, los rasgos sólo poseerían, de manera exclusiva, una función metalingüística y no sustancial.

Existe otra manera de determinar los rasgos semánticos. En este caso, hace referencia a la propiedad connotativa de las palabras. Se parte de la definición lexical o descriptiva del diccionario. Pero este método no ofrece mayor rigor ni seguridad que el anterior. Parece, más bien, que el camino correcto para el análisis componencial es el que trata de describir el significado de los términos que constituyen un campo semántico mediante los rasgos semánticos, obtenidos por comparación entre los lexemas y capaces de explicar el significado de todos los términos examinados en el campo semántico (Fernández González 1979: 146-8).

A manera de síntesis, se puede aceptar la afirmación que realizan varios autores a propósito de los principios de análisis componencial (Lyons 1989: Cap. 9: Sección 9). Estos principios serían los siguientes:

1. Principio de descomposición: el significado de cada palabra (o por lo menos el significado lógico-conceptual de cada palabra) puede descomponerse en una configuración o atado de rasgos semánticos. Según otra formulación, es representable mediante una configuración o atado de rasgos semánticos agrupados en conjunto para producir o representar ese significado. En consecuencia, la primera tarea del análisis componencial es la de proporcionar una descomposición o representación, mediante rasgos, de todas las palabras, de todo el léxico de una determinada lengua. La segunda se debe al hecho de que el léxico es tendencialmente abierto, o sea, está en constante renovación, pierde, y sobre todo, adquiere nuevos términos; está siempre en potencial expansión y mutación. Por eso, existen dos tipos de investigaciones parciales. La dirección descriptiva consiste en el análisis de rasgos de determinados campos semánticos o sectores del léxico (parentesco, colores, etc.). La dirección metateórica o meta descriptiva se refiere a los principios mediante los que puede realizarse el análisis de rasgos, cómo encauzar la dirección y cómo integrarla y caracterizarla dentro de la teoría general. En esta dirección, pierde importancia la descripción completa del léxico. Lo que interesa es el estatuto del análisis componencial y el modo en que explica las cosas; y resulta secundario que se tenga o no un análisis efectivo en rasgos de las palabras de la lengua. Este enfoque es propio de la gramática generativa.

2. Principio de economía y de generalización: pretende analizar el léxico sirviéndose de un número reducido de rasgos, muchos de los cuales aparecen en el significado de varias palabras. Se trata de manejarse con el menor número posible de elementos primitivos recurrentes, de manera que el significado de los varios millares de palabras de una lengua resulte de la combinación de algunos elementos tomados de un conjunto no demasiado extenso.

3. Principio de universalidad. Se considera que los componentes semánticos utilizados para describir el léxico de una lengua deben servir también para describir el léxico de otra; por lo tanto, los mismos componentes semánticos se vuelven a encontrar total o parcialmente, subyacentes en las distintas palabras de las distintas lenguas. Son campos semánticos homólogos con base en los mismos rasgos. 
4. Lyons incluye el principio de la relación del todo-parte. De acuerdo con este principio, no sólo existe una relación de jerarquía entre los lexemas sino de transitividad general basada en la redundancia; es decir, que ya el todo posee ciertos rasgos propios de las unidades. Sin embargo, las unidades en conjunto no sólo establecen relaciones entre sí, sino que producen un marco característico de significado que es propio sólo del todo y no de las distintas artes, pero que el todo de ningún modo podría poseer sino gracias a las características diferenciales de las partes individuales (Lyons 1989: 294-9).

A pesar de la claridad de estos principios, todavía persisten problemas no resueltos de manera totalmente satisfactoria. Entre ellos, el problema metodológico de la extracción de los rasgos, las técnicas empíricas utilizadas y la construcción de categorías analíticas. Sin embargo, no necesariamente un problema metodológico implica una neutralización epistemológica. Por esta razón, se puede suscribir la opinión siguiente:

\begin{abstract}
Los problemas y objeciones anteriores no impiden que existan óptimas razones para oponer a quien critica el análisis componencial (...) No existe ninguna prueba empírica de la universalidad de los componentes semánticos (y que tal vez en alguna lengua exista la posibilidad de que en el significado exista un rasgo que represente una característica de referente, obtenida por la experiencia. En tanto no repruebe lo contrario, los procedimientos de base lógica, fundados en las relaciones semánticas entre palabras (relaciones de semejanza y diferencia), cuyo reconocimiento, en cierta manera, debe integrar el bagaje lingüístico de los hablantes de una lengua, proporcionan un criterio suficientemente seguro para una primera extracción de los rasgos semánticos (Berruto 1974: 157-8).
\end{abstract}

En lo que se ha expresado anteriormente, se admitió el presupuesto de que las unidades léxicas mantienen un ámbito de significación definida o, por lo menos, restringida. Sin embargo, tanto la experiencia como los datos científicos demuestran que, en numerosas ocasiones, la ambigüedad y la imprecisión suelen ser más frecuentes de lo que se ha creído y que la descomposición en rasgos no es tan nítida como fuera deseable.

En 1924, Otto Jespersen, lingüista fuera del ámbito del estructuralismo pero con gran influencia en él, había reflexionado sobre el problema de construir una lingüística que diera cuenta de la movilidad y la inmovilidad del significado de las unidades lingüísticas, ya sean estas palabras, frases o grupos de palabras. En efecto, este autor dice:

\footnotetext{
Una expresión fija puede ser una frase o un grupo de palabras o una palabra o bien solamente parte de una palabra, poco importa, pero tiene que ser siempre algo que el instinto del habla siente como unidad imposible de analizar o de descomponer como en el caso de una expresión libre; pero, naturalmente, el tipo o modelo de acuerdo con el cual se crea una expresión libre tiene que estar vivo; así pues, las expresiones fijas pueden ser regulares o irregulares pero expresiones libres siempre presentan una expresión regular (Jespersen 1968: 17).
}

¿Qué es una expresión libre? La expresión libre es una unidad de significado con un referente como clase de señales o signos diferenciadores. Para el discípulo de Jespersen, Hjelmslev, la expresión enuncia un contenido y nunca está separada de tal. Son los signos con significación. Estos signos constituyen la base del análisis de los rasgos semánticos distintivos. El desplazamiento de significados amplía, distorsiona o enriquece, según se mire, la función significativa de los signos lingüísticos dentro del sistema de la lengua, debido, precisamente, a las relaciones que establece. Pero, ¿cómo opera el mecanismo de relaciones? 
Para explicarlo, debe verse la funcionalidad del mecanismo de transferencia de significado, según la cual una palabra sustituye a la habitual en un determinado significado; se funda en el parentesco de semejanza con base en la siguiente cadena: la palabra x utilizada correctamente para designar al referente x, es usada para designar al referente (Berruto 1974: 163). En términos generales, los cambios o desplazamientos de significado se pueden ubicar en las dos categorías del eje lingüístico: diacrónicas y sincrónicas. El modelo sigue siendo el clásico:

$$
\text { Sujeto } \frac{}{\text { referente }} \text { objeto }
$$

Ese modelo, aunque simple, es muy importante porque de ahí se derivan las siguientes consecuencias: a) existen afirmaciones con distinto significado pero que tienen el mismo referente; b) existen afirmaciones con significado pero que carecen de referente. Estas pueden ocurrir en palabras como sirena, centauro, etc. Son referencias vacías. Como es sabido, cuando la referencia es extensiva, entonces desempeña un papel destacado los prototipos $^{8}$. Pero cabe otro camino, que fue seguido por Leech. Según este autor, los rasgos semánticos en efecto enuncian una propiedad y construyen una representación semántica de los significados de las palabras y de las frases, trabajando en términos de predicado y argumento. Extendiendo este análisis a la frase, Leech la representa como constituida por tantos atados de rasgos como "grupos" que la forman. Esto permite superar el problema de las anomalías semánticas y de las restricciones selectivas; así como la polisemia y la ambigüedad. Con ello se pretende establecer una determinación de la clase significativa del rasgo y, a la vez, constituye una afirmación ontológica de la naturaleza del propio rasgo distintivo (Leech 1985: 166 y ss.).

En síntesis, cada palabra invoca e incorpora una carga de sentido y de significación que difumina la separación entre langue y parole, según la conocida terminología de Saussure, de manera tal que el contenido de cada palabra - y de cada oración - se combina con las contribuciones propias de su estructura interna y de su uso ${ }^{9}$. Es decir, que el sintagma (encadenamiento de palabras, serie regular) cuyo significante suele descomponerse en segmentos significativos, y el paradigma -que es más que un modelo flexivo- sino una realización de rasgos de oposición en un campo léxico, se convierten en determinantes sinsemánticos ${ }^{10}$.

Dicho de otro modo, tanto el sintagma como el paradigma son portadores de significación en tanto comparten una determinada referencia a un contexto y a una situación específica que se ubica dentro del sistema de manera estructurada, es decir, por medio de un proceso de interordinación semántica. De esta manera, se puede afirmar que, para la semántica estructural, los elementos constituyentes son sinsemánticos en la medida en que funcionan como operadores de significado de forma estructurada ${ }^{11}$.

Con lo expuesto anteriormente, se establecen dos respuestas que, aunque provisionales, son suficientes para el desempeño lingüístico de la semántica estructural. Por un lado, se admite la existencia objetiva de los rasgos dentro del sistema, independientemente del observador. Es la respuesta ontológica. Y por el otro, la posibilidad de establecer modelos estructurados sinsemánticos. Sin embargo, esto suscita otro problema, el referente a los límites de la estructura y, en consecuencia, a la determinación de su entorno lingüístico. 


\section{El significado según el análisis estructural}

Al integrar la dimensión formal con la semántica (interordinación), el modelo resuelve varios problemas. Uno de ellos es el de la pertinencia de los rasgos, el otro es el referido a la naturaleza de los rasgos y el tercero, el de la construcción de las matrices de rasgos. Lo interesante es que es una respuesta dentro de la semántica lingüística en sentido estricto sin necesidad de recurrir a categorías metafísicas o psicológicas. Por otra parte, en este análisis aparecen tres elementos: componentes semánticos, operadores (y, no, paréntesis), índices de referencia (X e Y con deponentes: los argumentos). Este proceso permite que los rasgos semánticos sean catalogados en dos clases: los rasgos predicativos, que representan propiedades y relaciones de los conjuntos de objetos a que nos referimos; y los rasgos delimitativos, que especifican a los conjuntos de objetos a que nos referimos con respecto a la cantidad y a las operaciones que conforman estos conjuntos.

Precisamente, el significado no puede partir de una idea mental, como había supuesto el mentalismo, sino de la consecuente interacción de signos lingüísticos. En otras palabras, la respuesta no puede limitarse a la operación de los signos de forma mecánica, aunque tampoco puede divagar sobre categorías metafísicas. Katz formuló la cuestión de forma decisiva:

\footnotetext{
Una teoría semántica debe explicar por qué el significado de una construcción lingüística la convierte en un caso de cierta relación o propiedad semántica, y hace que exponga el fenómeno de la sinonimia, la ambigüedad, la redundancia, etc. Por tanto, una respuesta a “QQué es el significado?” no puede obtenerse simplemente mediante la identificación del significado de la construcción lingüística con, digamos, aquello que nombra o a lo cual se refiere, o con unas disposiciones de acuerdo con las cuales es usado correctamente, o con la idea mental de la cual es el signo externo y sensible, o con estímulos sonsacadores y controladores que lo producen como respuesta verbal, o con el eterno arquetipo platónico al cual representa. (Katz 1979: 11, 483).
}

Afirmar esto implica tanto una restricción, porque cierra el ámbito explicativo dentro del entorno de la función sígnica, como una ampliación, porque vuelve flexible el modelo explicativo al abrir la posibilidad de la indeterminación de los signos dentro de una matriz definida. Para analizar las consecuencias de esta presuposición se comenzará por recordar varios conceptos importantes para la construcción del significado. Ellos son los siguientes: 1) cada uno de los elementos que forman la estructura guarda relaciones entre sí; 2) estas relaciones son de oposición; 3) suponen una distribución de los rasgos conceptuales; 4) implican la composicionalidad del signo lingüístico, ya que el significado se construye con elementos mínimos de significación; 5) ello implica una contextualización determinante; 6) de acuerdo con Greimas y Pottier, el significado siempre estará unido o derivado de relaciones léxico-sintagmáticas; 7) el significado categorial (Coseriu) en el cual los rasgos sémicos están en relación morfo-sintácticas y sus subdivisiones como designación distintiva de elementos de coherencia; 8) todo ello implica un proceso de interpretación referida a la interrelación de los significados con los símbolos lingüísticos dentro de un sistema axiomático abstracto, ya sea formal o informal del lenguaje natural; 9) este último punto implica la idea de un cálculo, entendido como un proceso deductivo a partir de reglas determinadas; 10) el cual, a su vez, implica la interordenación de elementos distintivos, donde la relación entre unidades normativas (sintaxis) y unidades significativas (semántica) se establece dentro de un paradigma a la vez ascendente y descendente; 11) este movimiento produce una jerarquía inmanente a la estructuración configuracional del significado ${ }^{12}$. 
En síntesis, todo significado es una interpelación que contiene una fórmula primitiva generada como asociación interdependiente basada en oposiciones, jerarquías y taxonomías, la cual expresa una funcionalidad linguiística, base de la interpretación semántica; es un proceso semántico de referencialidad categorial. En otras palabras, se admite el presupuesto saussureano de que todo sistema implica una jerarquía de relaciones entre los elementos, de tal modo que el significado se inscribe dentro de una totalidad determinada por un entorno. Pero los elementos de la totalidad interactúan entre sí para formar patrones de significación. La genial intuición de de Saussure aclaró que el valor del signo no es el significado y supuso un avance sobre los modelos anteriores. Esto implica que el significado de un término puede descomponerse en rasgos específicos (distinctive features), los cuales, a su vez, son diferentes según su relación con el significado (Alcaráz Varó 1997: 515). En efecto, al separar la función sígnica se puede establecer una autonomía relativa del nivel semántico. Sin embargo, el significado no reside sólo en una especie de ordenación de elementos. Supone algo más. ¿Qué es ese algo más del significado estructural?

El conjunto funciona como un operador semántico, es decir, como un aparato conceptual que establece la complejidad de los rasgos conceptuales, lo que permite un sistema de clasificación. En conjunto, el operador semántico forma una estimativa de significados posibles dentro de una red o matriz de rasgos interconectados. La construcción compleja de modelos explicativos con base en el método de composicionalidad estructural es llamada operador semántico. Este conjunto o sistema de signos lingüísticos relacionados entre sí permite establecer una estructura. Aunque la relación entre los signos es arbitraria, el significado proviene, precisamente, del sistema de la referencias internas de la estructura; en otras palabras, las relaciones entre los signos permiten determinar un significado específico. El operador semántico funciona determinando la estructura referencial interna de la lengua. Debe insistirse en que los elementos mínimos de significado, los semas, no son los elementos gramaticales morfo-sintácticos (sustantivo, verbo, adjetivo, afijos, suprasegmentales, etc.), ni tampoco la funcionalidad de los casos (nominativo, dativo, genitivo, etc.), sino las relaciones entre estas magnitudes sígnicas. No operan como el modi significandi medieval, pues excluye el hecho de que cada una de las partes de la oración o clases de palabras represente la realidad por medio de determinado modo o punto de vista. No es un modelo morfosemántico; más bien afirma la independencia del rasgo en dos niveles: el formal-estructural y el mental-representacional.

Como es sabido, la idea de un operador no es nueva. Se utiliza en lógica (operadores veritativo-funcionales, modales), en antropología (operadores totémicos, operadores sociales), en economía (operadores económicos), en ciencia política (operadores de decisión), en epistemología (operadores de necesidad epistémica), entre otros. En cada uno de los campos mencionados, el operador toma un valor particular no siempre coincidente. En esta tesis se afirma que los operadores semánticos permiten comprender mejor los procesos de significación semántica de las estructuras lingüísticas. Sin embargo, no debe entenderse este operador al modo de las gramáticas formales en las cuales suele ser considerado como un funtor, es decir, como una categoría que exprese la relación entre unidades del tipo: "y = F (x)", es decir, la propiedad "y" es concomitante a la propiedad " $x$ " de manera funcional. Sin embargo, esta es una concepción restringida. Más bien se debe aceptar una noción amplificada del operador en el cual su funcionalidad epistémico-metodológica no se reduce a lo semántico ya que tiene un ámbito propio de la lingüística. 
Para ser más precisos, considérese el siguiente caso. Existen dos semas distinguidos por sus relaciones de oposición (+), (-). Uno de ellos hace el papel de verificador (V), es decir, de afirmador de una característica (hombre, grande, rojo, etc.), y el otro de falsificador (de falsación)(F) de ese rasgo (no hombre, no grande, no rojo), lo que, a su vez, permite: (mujer, pequeño, verde, etc.).

El operador semántico establece y determina un juego o relación de partida en un enunciado dentro de un modelo M. De aquí se deriva una sucesión de enunciados que cumple las siguientes reglas básicas:

1. Si un enunciado es un elemento diferenciador de un rasgo binario, entonces el verificador establece el elemento dominante. En este caso, el rasgo verificador suele ser un cuantificador existencial (existe por lo menos un $\mathrm{x}$, tal que $\mathrm{x} . .$. ).

2. Pero en el caso de que el enunciado no forme parte de una fórmula dentro de $\mathrm{M}$, es decir, que sea un enunciado libre, entonces el falsificador establece la prioridad; en consecuencia, la disyunción es opcional, lo cual implica que el sema es un operador funcional dentro de $\mathrm{M}$.

No obstante, a diferencia de las formulaciones rígidas de la glosemática y sus seguidores, se puede considerar que el sema posee no sólo el rasgo diferenciador sino la relación de diferenciación, es decir, su carácter de verificador o de falsificador. De aquí surge una pregunta, ¿cómo se establece el rasgo? En este punto el formalismo estructural se muestra insuficiente. Se requiere otro factor fundante. Y este no puede ser un elemento dentro de la misma estructura, pues se caería en un círculo semántico cerrado en sí mismo. Parece que lo más adecuado sea recurrir a la diferenciación no formal, sino representacional, la cual opera como sustrato que hace posible la determinación de cada uno de los rasgos pertinentes.

Una consecuencia importante de la primera regla es que todas las relaciones entre semas son finitas, ya que cada uno de esos enunciados sólo permite un número finito de conexiones y de relaciones. Pero, aunque sean finitas, ¿cómo se sabe cuál relación es la adecuada, cuál es la "necesaria"? La respuesta a esta cuestión escapa o supera la estructura de la selección del rasgo porque también es siempre representacional y relacional. No es en sí misma formal. Una solución ha sido postular la existencia de un factor representacional sólo al inicio de la selección, pero excluirlo dentro del mecanismo de interrelación estructural. Este fue el camino seguido por la glosemántica. Sin embargo, esto pospone el problema, el cual vuelve a aparecer cuando se deben determinar los tipos de relaciones y las opciones posibles dentro del modelo.

En síntesis, el operador semántico incorpora las nociones de estructura, relaciones, semas, cálculo, interordinación, entre otros, para determinar los significados posibles dentro de una red o matriz semántica. No es un concepto lógico, sino más bien es un modelo funcional-semántico. $\mathrm{O}$, siendo más preciso, debe decirse que el operador semántico constituye un instrumento conceptual de aplicación analítica para determinar los valores semánticos tanto de los elementos, y las relaciones, como de la propiedad emergente considerada como significado. Derivado de ello, está el hecho de que el origen del significado se traslada de la superficie de los elementos lingüísticos (palabras, frases, oraciones) y sus funciones (verbo, sustantivo, adjetivo, etc.) o casos (nominativo, genitivo, dativo, etc.) para ubicarse en los elementos mínimos de la expresión, es decir, los semas. 
Sin embargo, debe recordarse que los semas no están en una función individual o independiente, sino en interrelación. Esto es, en el fondo, la estructura semántica, la cual establece un conjunto o totalidad de electos interrelacionados o interconectados de tal modo que establecen cierto tipo particular y específico de relaciones significantes. La modificación de los elementos o de las relaciones implica, necesariamente, la modificación de la estructura y, en consecuencia, el cambio de los elementos relacionales con el cambio de la significación lingüística. El operador semántico permite establecer las relaciones significativas que surgen de los elementos interrelacionados y la conformación de una unidad específica; unidad que determina el significado.

Se vuelve, pero por otro camino, a la teoría de de Saussure, quien afirmaba que la lengua (su significado) consistía en un sistema de signos interrelacionados. El significante y el significado, a pesar de que establecen una relación arbitraria entre sí, dan origen a que el significado del signo no existe por sí mismo sino dentro del sistema referencial interno de la propia lengua.

De esta forma, en el nivel semántico, es decir, el significativo, se establece una jerarquía diferente a la de los elementos que los forman, pero siempre dentro del plano lingüístico. Ello permite la elaboración de un modelo de significado formal pero no rígido ni mentalista. Esta teoría supone un campo semántico de interacciones mutuas. Lyons lo establece de la siguiente forma:

\footnotetext{
En lo que cabría considerar la más extrema versión de la teoría del campo, se establece que el vocabulario, V, de una lengua, es un conjunto cerrado de lexemas, $\mathrm{V}=\{\mathrm{L} 1, \mathrm{~L} 2, \mathrm{~L} 3, \ldots, \ln \}$, que pueden seccionarse en un conjunto de campos léxicos $\{\mathrm{C} 1, \mathrm{C} 2, \mathrm{C} 3, \ldots, \mathrm{Cm}\}$, es decir, divididos en subconjuntos, tal que (i) la intersección de los campos distintos cualesquiera es vacía (no hay ningún lexema que sea miembro de más de un campo), y (ii) la unión de todos los campos en $\mathrm{V}$ es igual a $\mathrm{V}$ (no hay ningún lexema que no pertenezca a algún campo) (Lyons 1989:152).
}

De acuerdo con esta versión, surge la posibilidad de que los campos sean abiertos e indeterminados; esto sin perjuicio de la formulación esencial básica del conjunto. Por tal motivo, la determinación del significado lingüístico no se circunscribe a la relación intersígnica. Más bien supone la indeterminación, el traslape o, incluso, la ausencia de elementos, los cuales forman parte de la noción de campo semántico. De ahí se deduce, además, que no necesariamente toda unidad del vocabulario pertenezca a un campo léxico y sólo a uno, así como puede existir la posibilidad de que no pertenezca a ninguno. Para aclarar este punto conviene dar un paso adelante.

En concordancia con la formulación realizada por el lingüista francés Greimas, la noción de significado también se puede entender como dentro de la configuración estructural de los componentes mínimos (semas). Esta caracterización permite analizar diversos sistemas sémicos o campos semánticos. Uno de ellos es el famoso análisis de la espacialidad o "campo semántico de la evaluación adjetival del espacio". Ej. Espacialidad: dimensionalidad-no dimensionalidad; horizontalidad-verticalidad, perspectividad-lateralidad, superficie-volumen, etc. (Greimas 1976: 50-2). La contribución de Greimas al tema de la significación lingüística es importante en la medida en que establece una precisión metodológica estricta, derivada de categorías lógico-formales. Ello permite determinar los "actores" de la narración como objetos del discurso y como posibles lexemas semiológicos. Sin embargo, el análisis de Greimas nos permite detectar las diferencias de cantidad y no sus antónimos. Además, existe el peligro de que se presenten términos redundantes: alto:vertical; lateral:horizontal, etc. 
A pesar de esta indeterminación, hay que mencionar varios conceptos. El primero de ellos es el de lexema, entendido como la unidad de contenido expresada en el sistema linguiístico. Son las unidades mínimas que corresponden al plano del significado; son lexemas los miembros de un campo léxico, es decir, las unidades funcionales de un campo léxico. Los lexemas se subdividen en simples y complejos. Estos últimos resultan de los procesos de producción semántica de las lenguas. El lexema es una unidad del plano de la lengua.

Por otra parte, el sema es el equivalente al fonón de la fonología. Es un eje significativo de expresión binaria y de oposición: [+ -]. En consecuencia, el semema es el conjunto de semas de un lexema; puede ser un archilexema, cuando son los lexemas que denotan un campo léxico. El archisema son los rasgos positivos en todos los campos léxicos. Siempre la unidad es el lexema. El archilexema constituye la unidad mayor; el hipolexema, la unidad menor; en ese caso la oposición se neutraliza (lobo, hombre, día: en genérico). El semema es el conjunto de semas; el archisema son semas constantes, los positivos en todos los campos. El clasema está formado por los semas contextuales.

Ampliando esta designación, Pottier, discípulo de Greimas, estableció que las lexías son los semas dentro de un entorno de relaciones binarias o multidireccionales pero que interactúan en conjunto con base en las oposiciones. Para aclarar este punto, a continuación se verá un ejemplo clásico propuesto por Pottier de una matriz de lexema (Pottier 1977: 64):

$\begin{array}{lcccccc}\text { "vehículo" } & \text { S1 } & \text { S2 } & \text { S3 } & \text { S4 } & \text { S5 } & \text { S6 } \\ \text { coche } & + & - & - & + & - & (+) \\ \text { taxi } & + & - & - & (+) & + & (+) \\ \text { autobús } & + & - & - & - & + & + \\ \text { autocar } & + & + & - & - & + & + \\ \text { metro } & + & - & - & - & + & + \\ \text { avión } & - & - & - & (+) & + & - \\ \text { moto } & + & - & + & - & - & (+) \\ \text { bicicleta } & + & - & + & - & - & (+) \\ \text { tren } & + & - & - & - & + & -\end{array}$

En este ejemplo se tienen los siguientes semas: S1 sobre el suelo; S2 sobre raíl; S3 dos ruedas; S4 individual; S5 de pago; S6 urbano. Los lexemas son: coche, taxi, autobús, etc. El archilexema corresponde a "vehículo"; el semema es el conjunto de semas; y el archisema es "para transporte". El signo + indica que comparte esta característica plenamente; por el contrario, el signo - denota que está exento de esa propiedad; y el paréntesis señala que ese sema específico no califica o que puede operar en ambos sentidos. El mismo autor añade que este análisis sémico de tipo binario puede ampliarse de forma múltiple. En este último caso, se podría utilizar un diagrama arbóreo. Sin embargo, la base de todo el sistema es siempre la estructura binaria de los componentes.

Ahora conviene dar un paso hacia delante. Al unir esta matriz con lo propuesto por Lyons se tiene lo siguiente: "V=\{L1, L2, L3 ... $\}$; o bien, $V=\{L 1, L 2, L 3$, etc. $\}$ y $C L=\{L i 1$, Li2, Li3... \}; o bien: CL 0 \{Li1, Li2, Li3, etc.” (Lyons 1989: 153), donde V se inscribe dentro de la matriz de rasgos pero con un peso sígnico indeterminado en sí mismo pero determinado dentro del sistema. Entonces, se tiene un operador semántico formalizado, es decir, una reconstrucción compleja de las relaciones que establecen el significado. 


\title{
4. ¿Descripción o interpretación?
}

Sin embargo, como precisa Katz, hay que distinguir entre una teoría semántica interpretativa y otra formal y descriptiva. Y esta distinción es importante porque incide en la determinación de los elementos significativos para la construcción semántica. De acuerdo con Katz:

\begin{abstract}
La cuestión entre las concepciones interpretativa y generativa es, pues, una controversia en torno a cuál de dos universales organizacionales debe ser incluido en la teoría lingüística. Por "universal organizacional" quiero significar una hipótesis que ordena los componentes de una gramática, unos con respecto a otros, y que proporciona direccionalidad en el proceso de generación de oraciones. Tales universales establecen que un componente A es anterior a un componente B, porque la información en el educto de A es necesaria para la operación de las reglas de B (...) Así, en este sentido, de direccionalidad, el componente transformacional es anterior al componente fonológico, en tanto que la información referente a la segmentación en la estructura patente es necesaria para la operación de reglas fonológicas (Katz 1979: 518).
\end{abstract}

En otras palabras, el modelo de la semántica implica un elemento dinámico de dirección (¿teleológico?); en consecuencia, no es sólo la estructura de la significación la que determina la interpretación posible; más bien, es la dinámica de los procesos sintáctico-semánticos los que definen el significado. Este modelo también parte de la determinación de rasgos distintivos: masculino-femenino-neutro, animado e inanimado, etc. La tendencia es a descomponer el significado. Pero aparece un problema, común al estructuralismo, debido al estatus de los términos que definen, porque al usar símbolos se cae en la circularidad en razón del uso de sistemas notacionales determinados en forma de cálculo diferenciado ${ }^{13}$. Aunque se trabaja con componentes del nivel léxico, también supone una unidad oracional. Se definen componentes nominales donde los símbolos complejos remiten a restricciones selectivas. Porque, si no aparecen definiciones anómalas en el nivel connotativo, se continúa con la investigación en el nivel de las estructuras profundas.

Por interpretación no debe entenderse la interpretación del texto, propio del análisis textual que versa sobre la descodificación de la unidad textual o enunciado dentro de un contexto (texto-contexto-pretexto). Más bien, debe entenderse como un proceso general y esencial de la lingüística. La interpretación semántica se refiere a la interrelación de los significados con los símbolos linguísticos dentro de un sistema axiomático abstracto, ya sea formal o informal del lenguaje natural; en otras palabras,

\footnotetext{
En el resultado de la aplicación de las reglas de proyección, el conjunto de indicadores sintagmáticos interpretados semánticamente, el output de una teoría semántica. La interpretación semántica debe caracterizar las ambigüedades semánticas, así como relacionar unas paráfrasis con otras, es decir, la capacidad de los hablantes para comprender las frases (Lewandowski 1982: 193).
}

Ya esta definición precisa dos elementos básicos. Por un lado, se refiere a la noción de reglas de proyección; es decir, al conjunto de normas que, según la teoría derivada de los trabajos de Chomsky, se proyecta tanto desde la estructura profunda a la superficial, así como en el movimiento que va de la sintaxis a la semántica. Por otro lado, también indica la función cognitiva del hablante para comprender las proferencias lingüísticas.

La lingüista I. Bellert ha estipulado el concepto de interpretación semántica al afirmar que la interpretación semántica de un enunciado, es decir, de un sintagma lingüísticamente definido, se refiere a "La serie de consecuencias que pueden derivar de ese enunciado (o, más estrechamente, de la descripción de su estructura profunda) sobre la base de ciertas reglas de implicación que se pueden establecer para ese lenguaje concreto" (En Lewandoski 1982: 193). 
Sin embargo, no debe pensarse que el concepto de interpretación se refiere únicamente a una estructura lógico-formal. Más bien la interpretación semántica se refiere tanto a las reglas de aplicación como al origen de las reglas, sus alcances y sus límites y su funcionalidad estructural.

Por otra parte, debe notarse que existe una diferencia con el modelo estructural clásico; a saber: aquí no se parte de un campo semántico o de oposiciones entre unidades de significado para describir la estructura paradigmática de los subsistemas léxicos. La descripción semántica de Katz tiende a constituir un léxico (diccionario) formado por una serie de entradas léxicas que representan el significado en cada lengua. De esta forma, cada entrada está compuesta por varios elementos: en primer término, la palabra como objeto, en su forma fonológica y ortográfica; luego, un conjunto de indicadores gramaticales o sintácticos; también, una o varias cadenas de símbolos, llamadas lecturas léxicas. A su vez, las lecturas léxicas están constituidas por un conjunto de símbolos llamados "indicadores semánticos” y por una restricción de selección (selection restriction). Los indicadores semánticos oponen la palabra en el objeto al resto del léxico; funcionan como rasgos oposicionales entre clases de palabras. Los distintivos semánticos oponen un sentido al otro de la misma palabra; funcionan como especificaciones que eliminan la ambigüedad: descartando un distintivo semántico, se eliminaría la posibilidad de anular la ambigüedad de uso de un término; descartando un indicador semántico, se deterioraría la estructura de todo el léxico.

Así, para Katz, el objetivo sobre el cual descansa su investigación del significado es integrar el tratamiento del significado de las palabras, analizando los rasgos semánticos, dentro de un tratamiento de la organización sintáctica de la frase. Para ello, establece varios puntos de partida: a) los juicios intuitivos que el hablante está en condiciones de dar respecto de los significados de la frase; b) el análisis de los elementos conceptuales y de las reglas de inserción que constituyen la posibilidad de uso de la palabra de la frase ${ }^{14}$. Con ello, la construcción del significado está constituida por los elementos propios de la oposición sígnica de los rasgos dentro del sistema, frente a un entorno y dentro de la función oracional. Ahora el problema se traslada de manera evidente al ámbito de la interpretación. Pero, bien mirado el problema, ¿no es la interpretación la base de todo el modelo de la semántica estructural?

Ahora el problema no es ni metodológico-epistemológico ni ontológico, se ha convertido en un problema hermenéutico. El modelo semántico estructural debe explicar la interpretación semántica que se da a los enunciados pre y post matriz de rasgos. Dicha interpretación resulta de la lectura posible de una frase, como combinación de las "lecturas" posibles de cada una de sus partes (es decir, de cada entrada del diccionario); en otras palabras, se ofrece una interpretación a los constituyentes gradualmente mayores (palabra, grupo, etc.) para llegar como resultado a la interpretación de la frase. Es, de nuevo, el complejo problema de la relación entre el todo y las partes (Greimas 1976: 43), problema que desemboca en la necesaria construcción de isotopías del discurso.

La noción de rasgo semántico desempeña una función importante en sus especificaciones de "indicador" y de "distintivo", puesto que se maneja con combinaciones reducidas del mismo conjunto de rasgos semánticos. Estos rasgos de transferencia constituyen una "condición para la denotación" (Weinreich), pues son fundamentales para constituir la oposición de los rasgos entre los signos; estos rasgos de transferencia se organizan dentro de conjuntos denotativos en una configuración cuando los rasgos se establecen con un orden de pertinencia; a su vez, toda la organización en su conjunto implica el significado. 
En consecuencia, el significado no podría ser el resultado solamente de un conjunto de elementos o rasgos semánticos unidos por constantes lógicas. Más bien, como se ha visto a propósito de la anterior caracterización de Lyons, una estructura E opera en un significado $\mathrm{S}$ cuando da como resultado un elemento más que la estructura $\mathrm{E}$; a este agregado dentro de la esfera de la acción de E, se le llama significado. Esquemáticamente: E (e+r) + E 1= S. Donde $e$ son los elementos constituyentes y $r$ son las relaciones; E1 es la propiedad emergente, no presente ni en los elementos individuales ni en las relaciones singulares; es siempre un valor emergente de la misma estructura, es decir, que no se encuentra en cada una de sus partes de manera aislada. El valor semántico de la estructura sería: E + S. Sin embargo, el proceso no es tan simple, pues, E es un agregado de elementos, de relaciones y de niveles. De igual manera, S contiene diversos niveles de significación. ¿Cómo determinar cuál es el apropiado? Precisamente aquí desempeña el papel el operador semántico, pues rige como una función, como cálculos interpretados.

Como se ha visto, el concepto de operador semántico se entiende como el conjunto de elementos que posibilitan las relaciones entre las partes de un universo semántico; por ejemplo, las oposiciones semánticas del tipo: hombre-mujer; joven-viejo y, en general, las que pueden clasificarse binariamente como positivo o negativo, o: 0,1 , las cuales constituyen la base de una matriz de rasgos. Los operadores pueden afectar los diferentes componentes de la oración, e, incluso, del texto. Es un constructo teórico inclusivo, es decir, supone elementos básicos, semas, dentro de unidades mayores. Así, por ejemplo, en el siguiente caso, "Juan es viejo", se supone la categoría "Hombre"; en el siguiente ejemplo: "María es bella", supone la categoría "Mujer". Pero, aún en el caso de una exclusión gramatical, el concepto propiamente semántico es inclusivo. Por ejemplo: "María solamente acostó a los niños" significa que se excluyen otras actividades que María pudo realizar, tales como, darles de comer, contarles un cuento, etc. (Alcaráz Martínez 1997: 391). Aunque el adverbio "solamente" opera sintácticamente como excluyente, en realidad incluye varios conceptos; es incluyente semánticamente.

Esta es una de la funciones más importantes del operador semántico: la de determinar los alcances conceptuales, representacionales mentalmente, de la semántica. De ahí que el concepto de oposición no sólo indica contraste, sino también establece una determinación categorial sobre los semas. Pues, como se ha visto, el sema, a la vez que excluye y determina una característica, siempre expresa una interordinación relacional. En el ejemplo anterior, "vehículo" opera como un signo integrador del cual surgen las características componenciales, analíticas y distribucionales, y no a la inversa. La estructura del operador semántico, es decir, del movimiento de integración, establece que la estructuración del significado surge, precisamente de estos movimientos: descomposición, distribución e integración.

En esto se diferencia de las oposiciones fonológicas que son esencialmente privativas, bilaterales, correlativas o disyuntivas. En el caso de las oposiciones semánticas, el estructuralismo ha demostrado que son, más bien, de naturaleza inclusiva, conjuntivas, multilaterales. Nunca podrían ser privativas ni aisladas, es decir, que se producen únicamente en el seno de una matriz de rasgos determinada previamente, dentro de la cual adquieren un haz de significaciones.

Por otra parte, es importante recordar que I. Bellert ha propuesto el concepto de operador referencial. Se utiliza para las descripciones indefinidas pero que poseen referencias particulares: “(x) F(x)". Sin embargo, es del mismo tipo de operadores existenciales propios de 
los enunciados existenciales del tipo: "existe un; o por lo menos un". Pero con ello se reduce el campo aplicativo (En Lewandowski 1982: 248). Más importante ha sido la propuesta de Carnap del Operador Lx, pues, "en este caso tiene la función específica de conformar una expresión para toda propiedad adscrita a un individuo por una oración en una lengua" (Lewandowski 1982: 248). El operador establece una serie de relaciones semántico-gramaticales del tipo determinans-determinatum; pero, a diferencia de los operadores antes mencionados, el operador semántico siempre establece un significado dentro de una matriz de rasgos conformada por el procedimiento de la composicionalidad estructural.

El sentido de los operadores semánticos se deriva del conjunto de rasgos relacionales dentro de un proceso de composicionalidad. En la semántica estructural, el sentido operativo semánticamente articulado es eidético, es decir, supone siempre una referencialidad extrasígnica y no sólo formal. En este aspecto, y extrapolando su valor denotativo, se puede aceptar la opinión que ofrece el lingüista alemán Werner Abraham al afirmar lo siguiente:

\footnotetext{
Decimos que un signo tiene en un sistema de signos un sentido eidético, cuando sabemos qué designa ese signo, o bien, cuando al menos sabemos que designa algo. Las cosas se presentan de otra manera cuando no conocemos tal objeto y sabemos únicamente cómo hay que operar con ese signo. (De aquí el término, "sentido operativo"). Y no es que corresponda esta descripción sólo a signos de lenguajes, cálculos matemáticos o lógicos construidos artificialmente. Naturalmente estos lenguajes son el campo de acción característicos de sistemas de signos con sentido predominantemente operativo. Un cálculo matemático operativo frecuentemente no tiene en principio ningún sentido eidético (Abraham 1981: 328).
}

En otras palabras, el sentido operativo de un signo dentro del modelo estructural es siempre eidético, ya que se presume la existencia de un designatum. No es, en modo alguno, un operador formal carente de referencialidad. Es importante recalcar este punto, pues en varias ocasiones se ha querido establecer una referencialidad cero o vacía de los elementos semánticos. Quizás el caso más extremo haya sido el de Hjelmslev; sin embargo, la suprema abstracción de este modelo semántico tampoco ha excluido los elementos eidéticos.

No es sólo un isomorfismo entre lenguaje y pensamiento; es, más bien, una solidaria fusión de la intersubjetividad personal y social. La inteligibilidad intrínseca al sujeto requiere el dinamismo del lenguaje del sujeto (Cortés 1994: 313). El proceso semántico implica una determinación de la referencialidad categorial. Este problema es particularmente sensible cuando uno de los participantes en el proceso comunicativo tiene alterada o reducida la capacidad de descodificación simbólica, como es el caso de personas con discapacidad léxica o gramatical (agramatismo), afasia, retardo mental o algún trauma neurológico, etc. De ahí que el interés sea tanto el de aumentar el conocimiento de la ciencia lingüística (función teóricocientífica), como el de resolver un problema concreto (función técnico-aplicativa), pues se ha detectado que en numerosas ocasiones existe una discrepancia lingüística y un ruido cognitivo que interfiere y distorsiona los procesos y resultados de la comprensión comunicativa.

Además, recuérdese la noción de significado estructural propuesta por Greimas. Vista desde la idea del operador semántico, funciona como una propiedad emergente de la totalidad estructural, pero siempre dentro de una matriz de rasgos semánticos. Dentro de la estructura configurada por el análisis composicional, el significado tiende a ser determinado por los elementos que la componen pero sin que se restrinja a una simple suma de elementos. Todo lo contrario, el análisis sémico de estas unidades estructuralmente determinadas ciertamente supone en una primera instancia la descomposición en rasgos semánticos, pero, además, implica que existe un cálculo, es decir, un modelo deductivo que contiene reglas, 
signos, axiomas como parte constituyente del todo semántico. En el ejemplo antes mencionado de Pottier se aprecian claramente las tres funciones: la distribucional propia de la diferenciación de los rasgos semánticos, la distribucional, correspondiente a las categorías jeraquizadas (interordinadas), y la de la integración, que surge de las anteriores y es la que permite establecer el significado.

\section{Observaciones finales}

En este artículo se ha realizado una exposición de las características más relevantes de la semántica estructural. Especial atención ha recibido la noción de rasgo semántico, de campo y de análisis composicional.

La complejidad de la estructura, sus notas distintivas y sus consecuencias lógico-formales han sido establecidas merced a la intervención de un elemento teórico que se ha designado como operador semántico, el cual es un constructo de naturaleza heurística que permite establecer las relaciones formales dentro de una matriz de rasgos, y a la vez, establecer los enlaces pertinentes para el proceso de interpretación tanto de los rasgos como de las matrices semánticas. Como se vio al principio, además existe un movimiento de jeraquización de rasgos dentro del todo. Es el llamado de interordinación, o sea, la construcción de un significado donde la relación entre unidades normativas y unidades significativas establecida en rasgos se formula en un doble movimiento a la vez ascendente y descendente. Este movimiento produce una jerarquía inmanente a la estructuración configuracional del significado. Toda oración es una interpelación, es decir, que contiene una fórmula primitiva generada como asociación interdependiente. Por eso, el modelo estructural basado en oposiciones, jerarquías y taxonomías expresa una funcionalidad lingüística, base de la interpretación semántica. Es lo que Coseriu había llamado significado categorial. Aquí los lexemas tienen tanto un significado léxico como uno categorial. Este es el movimiento que establece el significado desde la estructuración integrada de cada miembro de un enunciado. Es una consecuencia de la noción de campo asociativo o campo léxico, de tal manera que el cálculo semántico es un presupuesto implícito de la relación entre los diversos rasgos significantes del análisis distribucional. Ahora se comprende porqué en el análisis componencial existe, necesariamente, una reducción de los elementos constituyentes a sus partes mínimas, los cuales aparecen en una unidad lingüística. En consecuencia, la composicionalidad del signo lingüístico opera debido a que el significado se establece no sólo con base en los rasgos de oposición, como elementos exclusivos y excluyentes; más bien, son elementos operatorios inclusivos. Es decir, que los rasgos de oposición no son diferenciadores, sino integradores del sentido.

Sin embargo, a diferencia de lo que postula la teoría clásica de la semántica estructural (Greimas, Coseriu, Berruto, etc.), el operador semántico permite distinguir, además de las funciones tradicionales, la distribucional y la componencial, una función diferente. Esta es la función de integración semántica. Sin la existencia de esta función no podría producirse el significado linguiístico. De tal modo que los semas y sememas actúan distribucional, componencial e integradoramente.

Derivado de lo anterior, pero en un plano diferente, es apropiado afirmar que el lenguaje no es tanto el vehículo del pensamiento sino, más bien, el medio condicionante de este; es decir, un mecanismo de construcción cognitiva sobre una base categorial. Porque el lenguaje 
posee una función de la mayor importancia, a saber: condicionar el pensamiento, el sentimiento y hasta el modo de actuar. ${ }^{15}$ La semántica estructural, que parece ser una metodología rígida y formal, en realidad permite una visión dinámica de la lengua en su función significante.

Asimismo, se ha reconstruido el modelo de la semántica estructural sobre la base de su aplicabilidad funcional. Con la introducción del operador semántico, se establecen tres categorías funcionales básicas, y no dos, como era usual en la teoría clásica: la distribucional, la componencial y la de integración. No es fácil llegar a una conclusión definitiva. En efecto, este modelo, más que un todo acabado, aparece como un extenso proceso de investigación semántica que aún no ha concluido. Es un proyecto que, con sus luces y sombras, mantiene su vigencia y todavía puede aportar mucho más a la investigación del significado linguiístico. Por eso, al tratar con el lenguaje no debería olvidarse el proceso de recursión, pues el lenguaje habla del lenguaje - de sí mismo—-, lo crea, lo estructura y lo determina. De esta forma, se entiende que las funciones comunicativas, adaptativa y cognitiva se integran en la determinación del conocimiento dentro de un proceso de estructuración de la expresión.

Quizás tuviera razón Humboldt cuando hablaba de la energía inherente al lenguaje; de la dynamis del decir; fuerza o energía que, precisamente, han querido atrapar los lingüistas: el mismo Humboldt, de Saussure, cualquiera de los grandes estudiosos del lenguaje y que la realidad demuestra una parcialidad cómplice con el exceder y el superar. Es sabido que no existe ningún sistema que aprese al lenguaje; pero, por otra parte, sin sistema el lenguaje sería incomprensible. Decir y no decir, palabra y silencio, es la dinámica del lenguaje y su conocimiento es la tarea — ¿imposible? — del lingüista.

\section{Notas}

1. Los rasgos semánticos también han sido llamados rasgos componentes, semas, semema, indicadores, rasgos pertinentes, figuras, diferenciadores, etc.

2. Las expresiones espaciales tales como "lugar", "arriba", "abajo", "ascendente", "descendente", entre otras, constituyen un uso metafórico para referirse al ámbito semántico exclusivamente.

3. Como se puede observar, debido a la propia naturaleza de los rasgos, estos no agotan otros aspectos como los elementos estilísticos y combinatorios, o de registro expresivo, etc., que hacen referencia ya sea a la estilística, la sintaxis o la pragmática.

4. Como se puede apreciar, la estructura de fondo del procedimiento se fundamenta en la teoría de conjuntos. Tanto que podría hablarse de una función de conjunto de los lexemas.

5. La valoración que aquí se realiza difiere de la de Berruto, para quien estos modelos tienen la ventaja de que el significado de una expresión compleja (una frase) ya no es visto solamente como una masa no estructurada de rasgos (de la misma manera en que era visto el significado en una palabra), potencialmente incapaz de explicar los muchos caso de ambigüedad, dado que en aquella teoría si dos frases estaban compuestas por las mismas palabras, aún si estaban colocadas en distinto orden, debían estar compuestas por la misma masa de rasgos. Porque, visto de esta forma, el problema del significado lingüístico todavía no ha sido resuelto satisfactoriamente.

6. Este es el motivo por el cual las características sintácticas y estilísticas son la causa de que la sinonimia perfecta no exista, sino en casos excepcionales. Precisamente, dos palabras del mismo grupo semántico pueden tener por lo menos un rasgo sintáctico, estilístico o pragmático diferente. Los rasgos pragmáticos 
son importantes porque unen la estructura lingüística con su uso por parte de la comunidad de hablantes y con las variaciones que sufre la lengua dentro de las concretas exigencias y realizaciones comunicativas de una sociedad.

7. Dentro de este modelo explicativo se puede decir que la sinonimia es el hecho de que dos lexemas están constituidos por el mismo conjunto de rasgos semánticos. En cambio, la hiponimia corresponde al conjunto de rasgos semánticos de una palabra, el cual está totalmente comprendido en el conjunto de rasgos semánticos de otra palabra. La incompatibilidad (antonimia, complementariedad, inversión) es el hecho de que el significado de una palabra contiene un rasgo opuesto al contenido en el significado de otra.

8. La semántica de los prototipos ha sido desarrollada por Kleiber, para quien un prototipo vendría ser una entidad relacionada con la representación de la extensión de un objeto (Kleiber 1995).

9. Como ya había demostrado Kant, las oraciones pueden poseer varios tipos de propiedades de sentido y de valor de verdad. Las primeras son las analíticas, que siempre son verdaderas en virtud de su forma lógico-sintáctica, porque el significado del sujeto contiene las propiedades denotadas por el predicado. Kant las denominó juicios analíticos. Siempre son válidas. En cambio, en las sintéticas, la verdad o falsedad depende siempre de circunstancias o hechos extralingüísticos. En este caso, su verdad es de tipo referencial, siempre, porque el significado del sujeto no está incluido dentro del predicado. El último grupo son las contradicciones, que siempre serán falsas, ya que las propiedades del significado del sujeto contradicen la propiedad denotada en el predicado (Kant 1985).

10. Entendido como: "Portador (potencial) de significado (palabras, morfemas) que sólo son significativas en la composición o combinación con otros portadores de significado” (Lewandowski 1982: 322).

11. Se nota que los lingüistas estructurales de la llamada primera etapa (Trier, de Saussure, Hjelmslev, Benveniste, Coseriu, Greimas, etc.) suponen una estructura o sistema cerrado donde operan los rasgos distintivos y el universo de oposiciones binarias. En cambio, los de la segunda etapa (Lyons, Berruto, Geckeler, etc.) admiten que la lengua actúa como un sistema abierto. Esto les permite establecer diversas conexiones con otros elementos del análisis lingüístico dando lugar a un modelo mucho más dinámico y funcional. Para la presente investigación, este último modelo es más indicado porque implica que los rasgos de oposición funcionan como signos sinsemánticos y, a la vez, como operadores abiertos, pero no exentos de sentido.

12. Al precisar mejor esta caracterización, se tiene el siguiente resumen de los elementos esenciales de la semántica estructural, a saber: a) un conjunto de elementos diferenciados y diferenciadores gracias a la función de rasgos específicos (semas); b) un conjunto de elementos diferenciadores por el procedimiento de semejanzas y diferencias formales y funcionales (oposición), por medio de una interrelación de los elementos constitutivos; c) una estructura o sistema constituido por tales redes de asociaciones (matriz de rasgos: +, -); d) una interrelación de los elementos constitutivos por unidades (lexemas) en un campo semántico; e) la diferenciación de los elementos (su oposición) es la que determina su singularidad lingüística; f) una serie de características originales del sistema, que Lyons llama "terminales sin existencia previa", las cuales permiten establecer las unidades de semejanza-diferenciación dentro del propio sistema; g) de tal conjunto de asociaciones se deriva (se compone) el significado y la significación semántica dentro del modelo lingüístico; h) en una primera etapa, la semántica se derivaba exclusivamente de la diferencia de los rasgos léxicos (gramaticalización reductiva), de tal modo que el significado era el resultado de patrones de oposición colocados en una matriz de rasgos.

13. Es el problema de la circularidad en el uso de los signos: un signo define otro signo y así sucesivamente. Pero, en este caso, los rasgos establecen un correlato con los signos o marcadores sintácticos que rompen el círculo conceptual.

14. Para Berruto, los indicadores vacíos X e Y señalan, respectivamente, las posiciones en que deben encontrarse el sujeto y el objeto que indican la restricción selectiva. Estas restricciones selectivas coinciden con 
las "reglas selectivas", que según Chomsky debería, más bien, formar parte de la sintaxis y no de la semántica; en términos de la gramática generativo-transformacional, deberían formar parte del componente sintáctico y no del componente semántico. Sería, por ejemplo, una regla selectiva la que asigna a "frighten" (asustar) el rasgo (+(+abstracto)- - -(+animado)) (Berruto).

15. Una contribución clásica ha sido la de G. W. Leibniz. 1977. Nuevos ensayos sobre el entendimiento humano. Libro III: Sobre las palabras. Madrid: Editora Nacional. En este caso el racionalismo implica una necesaria toma de posición sobre el origen de las categorías.

\section{Bibliografía}

Abraham, Werner. 1981. Diccionario de terminología lingüística actual. Madrid: Gredos.

Alarcos Llorach, Emilio. 1990. Gramática estructural según la Escuela de Copenhague y con especial atención a la lengua española. Madrid: Gredos.

Alcaráz Varó, E. y M. Martínez Linares. 1997. Diccionario de lingüística moderna. Barcelona: Ariel.

Alonso-Cortés, A. 1993. Lingüística general. Madrid: Cátedra.

Arens, Hans. 1976. La lingüística. Sus textos y evolución desde la antigüedad hasta nuestros días. (Dos vols.) Madrid: Gredos.

Bellert, I. 1969. Arguments and predicates in logic-semantics structures of utterance. En: Lewandoswski: 1982.

Benveniste, Émile. 1985. Estructura en lingüística. En: É. Benveniste, Problemas de lingüística general. México, Siglo XXI.

1985. Problemas de lingüística general. México: Siglo XXI.

Berruto, G. 1974. La semántica. México: Nueva Imagen.

Bierwisch, M. 1971. El estructuralismo. Historia, problemas, métodos. Barcelona: Tusquets.

1976. Sobre la clasificación de los rasgos semánticos. En: John Lyons. Nuevos horizontes en la lingüística. Madrid, Alianza Editorial, 1975.

Chomsky, Noam. 1999. Aspectos de la teoría de la sintaxis. Barcelona: GEDISA, S. A.

1974. Estructuras sintácticas. México: Siglo XXI.

1984. Lingüística cartesiana. Madrid: Gredos. 
1998. On Language. Language and Responsability. Reflections on Language. Nueva York: The New Press.

Coseriu, Eugenio. 1987. Gramática, semántica, universales. Madrid: Gredos.

1977. Principios de semántica estructural. Madrid: Gredos.

1982. Teoría del lenguaje y lingüística general. Madrid: Gredos.

Crystal, David. 1997. The Cambridge Encyclopedia of Language. Cambridge University Press.

Dubois, Jean, et al. 1999. Dictionnaire de linguistique et des sciences du langage. París: Larousse.

Ducrot, Osvald. 1982. Decir y no decir. Principios de semántica lingüística. Barcelona: Anagrama.

Ducrot, Osvald y T. Todorov. 1985. Diccionario enciclopédico de las ciencias del lenguaje. México: Siglo XXI Editores.

Fernández Pérez, M. 1999. Introducción a la lingüística. Barcelona: Ariel, S. A.

Geckeler, Hans. 1994. Semántica estructural y teoría del campo léxico. Madrid: Gredos.

Greimas, A. J. 1976. Semántica estructural. Investigación metodológica. Madrid: Gredos.

Hjelmslev, Louis. 1978. La teoría de los casos. Madrid: Gredos.

1971. Prolegómenos a una teoría del lenguaje. Madrid: Gredos.

Hurford, J. R. y B. Heasley. 1983. Curso de semántica. Madrid: Visor.

Jakobson, Roman. 1984. Ensayos de lingüística general. Barcelona: Ariel.

Jespersen, Otto. 1975. La filosofía de la gramática. Barcelona: Anagrama.

Kant, Inmanuel. 1985. Crítica de la razón pura. Madrid: Alfaguara.

Katz, J. 1979. Teoría semántica. Madrid: Aguilar.

Katz, J. y J. A. Fodor. 1963. “The structure of a semantic theory.” Language. 39 (2): AprilJune.

Kleiber, Georges. 1995. La semántica de los prototipos. Categoría y sentido léxico. Madrid: Visor Libros. 
Koerner, E. F. 1982. Ferdinand de Saussure. Génesis y evolución de su pensamiento en el marco de la lingüística occidental. Madrid: Gredos.

Ladusaw, W. 1990. Teoría semántica. En: Newmeyer, F. J. (Comp.) 1990. Panorama de la lingüística moderna de la Universidad de Cambridge. Madrid: Visor, S. A.

Leech, Geoffrey. 1985. Semántica. Madrid: Alianza Editorial.

Leibniz, G. W. 1977. Nuevos ensayos sobre el entendimiento humano. Madrid: Editora Nacional.

Lewandowski, Theodor. Diccionario de lingüística. Madrid: Ediciones Cátedra, S.A.

Lyons, John. 1974. Chomsky. Barcelona: Grijalbo, S. A.

1983. Lenguaje, significado y contexto. Barcelona: Paidós Ibérica, S. A.

(Ed.). 1975. Nuevos horizontes de la lingüística. Madrid: Alianza Editorial.

1989. Semántica. Barcelona: Editorial Teide.

1997. Semántica lingüística. Una introducción. Barcelona: Paidós Ibérica, S. A.

Marín, Francisco. M. 1994. Introducción a la lingüística: historia y modelos. Madrid: Síntesis.

Martinich, A. P. (Edit.). 1990. The Philosophy of Language. Nueva York-Oxford: Oxford University Press.

Mounin, Georges. (Dir). 1979. Diccionario de lingüística. Barcelona: Labor, S. A.

1984. La lingüística del siglo XX. Madrid: Gredos.

1971. Saussure. Presentación y textos. Barcelona: Anagrama.

Newmeyer, F. (Comp.). 1990. Panorama de la lingüística moderna de la Universidad de Cambridge. Madrid: Visor, S. A.

Ogden, C. K. y I. A. Richards. 1984. El significado del significado. Una investigación acerca de la influencia del lenguaje sobre el pensamiento y de la ciencia simbólica. Barcelona: Paidós.

Pottier, Bernard. 1976. Lingüística general. Teoría y descripción. Madrid: Gredos.

Robins, R. H. 1995. Lingüística general. Madrid: Gredos. 
Rodríguez Adrados, Francisco. 1974. Lingüística estructural (Dos vols.) Madrid: Gredos.

Saussure, Ferdinand de. 1983. Curso de lingüística general. (Trad. Amado Alonso). Madrid: Alianza Editorial.

Trujillo, Ramón. 1979. Elementos de una semántica lingüística. Madrid: Cátedra.

Tusón, Jesús. 2000. ¿Cómo es que nos entendemos? (si es que nos entendemos). Barcelona: Península.

Ullmann, S. 1967. Semántica. Introducción a la ciencia del significado. Madrid: Aguilar.

Vilarnovo, A. 1993. Lógica y lenguaje en Eugenio Coseriu. Madrid: Gredos.

Weinreich, H. 1968. Estructura y función de los tiempos en el lenguaje. Madrid: Gredos. (Edit.) 1981. Lenguaje en textos. Madrid: Gredos.

Weisgerber, Leo. 1979. Dos enfoques del lenguaje. Lingüística y ciencia energética del lenguaje. Madrid: Gredos.

Wotjak, Gerhardt. 1979. Investigaciones sobre la estructura del significado. Madrid: Gredos.

Yule, George. 1998. El lenguaje. Barcelona: Visor. 